\title{
Thyroid Hormone Improves Function and $\mathrm{Ca}^{2+}$ Handling in Pressure Overload Hypertrophy
}

\author{
Association with Increased Sarcoplasmic Reticulum $\mathrm{Ca}^{2+}$-ATPase and $\alpha$-Myosin Heavy Chain in Rat Hearts
}

\author{
Kevin C. Chang, ${ }^{\S}$ Vincent M. Figueredo, ${ }^{* \|}$ Joop H.M. Schreur, ${ }^{*}$ Ken-ichi Kariya, ${ }^{\S}$ Michael W. Weiner, ${ }^{\star \star \S}$ Paul C. Simpson, ${ }^{\star \S}$ \\ and S. Albert Camacho*\| \\ *Department of Medicine, University of California, San Francisco, California 94143; ${ }^{\ddagger}$ Department of Radiology, University of California, \\ San Francisco, California 94143; ${ }^{\S}$ the Research Service, Department of Veterans Affairs Medical Center, San Francisco, California 94121;

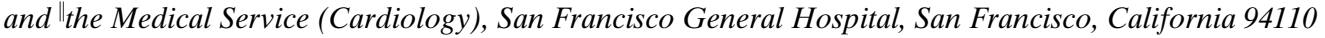

\begin{abstract}
We asked whether thyroid hormone $\left(\mathrm{T}_{4}\right)$ would improve heart function in left ventricular hypertrophy (LVH) induced by pressure overload (aortic banding). After banding for 10-22 wk, rats were treated with $T_{4}$ or saline for 10-14 d. Isovolumic LV pressure and cytosolic $\left[\mathrm{Ca}^{2+}\right]$ (indo-1) were assessed in perfused hearts. Sarcoplasmic reticulum $\mathrm{Ca}^{2+}$ ATPase (SERCA), phospholamban, and $\alpha$ - and $\beta$-myosin heavy chain (MHC) proteins were assayed in homogenates of myocytes isolated from the same hearts. Of 14 banded hearts treated with saline, 8 had compensated LVH with normal function $\left(\mathrm{LVH}_{\text {comp }}\right)$, whereas 6 had abnormal contraction, relaxation, and calcium handling $\left(\mathrm{LVH}_{\text {decomp }}\right)$. In contrast, banded animals treated with $\mathrm{T}_{4}$ had no myocardial dysfunction; these hearts had increased contractility, and faster relaxation and cytosolic $\left[\mathrm{Ca}^{2+}\right]$ decline compared with $\mathbf{L V H}_{\text {comp }}$ and $\mathbf{L V H}_{\text {decomp. }}$. Myocytes from banded hearts treated with $\mathrm{T}_{4}$ were hypertrophied but had increased concentrations of $\alpha-\mathrm{MHC}$ and SERCA proteins, similar to physiological hypertrophy induced by exercise. Thus thyroid hormone improves LV function and calcium handling in pressure overload hypertrophy, and these beneficial effects are related to changes in myocyte gene expression. Induction of physiological hypertrophy by thyroid hormonelike signaling might be a therapeutic strategy for treating cardiac dysfunction in pathological hypertrophy and heart failure. (J. Clin. Invest. 1997. 100:1742-1749.) Key words: contractility - myocardial relaxation $\cdot$ pathological hypertrophy $\bullet$ fluorescence $\bullet$ indo-1 AM
\end{abstract}

Preliminary results of this study were presented at the 68th Annual Scientific Session of the American Heart Association in Anaheim, CA on 13-16 November 1995 and have appeared in abstract form (1995. Circulation. 92[8]:I-255).

Address correspondence to S. Albert Camacho, Division of Cardiology, Room 5G1, UCSF/San Francisco General Hospital, San Francisco, CA 94110. Phone: 415-206-8862; FAX: 415-206-5100; E-mail: sacama@itsa.uscf.edu Kevin Chang's current address is Department of Medicine, University of Nevada, Reno, NV. Joop H.M. Schreur's present address is Rode Kruis Ziekenhuis, The Hague, The Netherlands. Ken-ichi Kariya's current address is Department of Physiology II, Kobe University School of Medicine, Japan.

Received for publication 17 June 1996 and accepted in revised form 14 August 1997.

The Journal of Clinical Investigation

Volume 100, Number 7, October 1997, 1742-1749

http://www.jci.org

\section{Introduction}

The importance of heart failure as a clinical problem emphasizes the need to develop novel treatment strategies (1). One approach focuses on myocardial hypertrophy, an invariant pathological feature in the failing heart. Myocardial hypertrophy is a compensatory response to the increased load imposed by hypertension, valve disease, or myocardial infarction. However, function in the hypertrophied heart eventually becomes abnormal, the so-called transition from hypertrophy to failure $(2,3)$. Because function eventually becomes abnormal, the overall growth process in the setting of cardiac disease has been called pathological hypertrophy (4).

Physiological processes such as exercise and development also cause myocardial hypertrophy, but myocardial function is normal or enhanced (4-6). Although the molecular mechanisms underlying physiological versus pathological hypertrophy remain uncertain, one potential therapeutic strategy might be to convert a pathological growth process to a physiological one. Indeed, Scheuer and colleagues showed that swimming exercise improved cardiac function in rats with pressure overload hypertrophy, even though heart weight was increased (7-9).

Thyroid hormone has the potential to mimic the ability of exercise to convert pathological to physiological hypertrophy. Thyroid treatment of normal animals induces a physiological hypertrophy characterized by faster myocardial relaxation and decline of the cytosolic calcium transient (10-13). Recently, thyroid treatment was shown to improve myocardial function in the postinfarction model of heart failure (14-17). However, thyroid hormone may enhance cardiac function in the postinfarct model partly by decreasing vascular resistance $(13,16)$. Furthermore, the physiology and gene expression of noninfarcted myocardium in the postinfarct model may differ from pressure overload models of pathological hypertrophy.

We asked whether thyroid hormone $\left(\mathrm{T}_{4}\right)$ would improve myocardial function in a model of pathological hypertrophy induced by chronic, sustained pressure overload. Because pressure overload was produced by banding of the aorta, vascular effects of thyroid hormone were minimized. After 10-22 wk of banding, animals were randomized to receive thyroid hormone or vehicle for 10-14 days. Left ventricle (LV) ${ }^{1}$ func-

1. Abbreviations used in this paper: $\tau_{\mathrm{Ca}}$, time constant of the exponential decline of the $\left[\mathrm{Ca}^{2+}\right]$ transient; KH, Krebs-Henseleit; LV, left ventricle; LVDevP, left ventricular developed pressure; LVH, LV hypertrophy; $\mathrm{LVH}_{\text {comp }}$, compensated $\mathrm{LVH} ; \mathrm{LVH}_{\text {decomp }}$, decompensated LVH; MHC, myosin heavy chain; $\tau_{\mathrm{p}}$, time constant of the exponential decline of LV pressure transient; SERCA, sarcoplasmic reticulum $\mathrm{Ca}^{2+}$-ATPase. 
tion and cytosolic $\left[\mathrm{Ca}^{2+}\right]$ (indo-1) were assessed in isovolumic perfused hearts. Myocytes were isolated from these same hearts to quantify important calcium handling and myofilament proteins: sarcoplasmic reticulum $\mathrm{Ca}^{2+}$-ATPase (SERCA), phospholamban, and the $\alpha$ - and $\beta$-myosin heavy chain (MHC) isoforms. Studies in many models have found a general correlation between induction of $\alpha$-MHC and SERCA and a more physiological hypertrophy $(4,5,12,18)$. However, it is not known whether SERCA and $\alpha$-MHC can be upregulated in the presence of persistent pressure overload.

We report that thyroid hormone improves LV function and calcium handling in the model of chronic, sustained pressure overload, and that the functional benefit is related to changes in the expression of SERCA and $\alpha$-MHC in cardiac myocytes. We conclude that induction of physiological hypertrophy by thyroid hormone-like signaling in myocytes should be considered as a therapeutic strategy for treating cardiac dysfunction in pathological hypertrophy and heart failure.

\section{Methods}

Animal preparation. Pressure overload was created in male rats (Sprague-Dawley, 200-220 grams; Simonsen Co., Gilroy, CA) by banding of the aorta above the renal arteries (19). Either a Weck hemoclip (Edward Weck \& Co., Inc., Research Triangle Park, NC) or a silk suture were used to reduce internal diameter of the aorta to 0.6 $\mathrm{mm}$ for 20-22 wk $(n=5)$ or to $0.3 \mathrm{~mm}$ for $10-12 \mathrm{wk}(n=9)$. These two different banding protocols induced equivalent degrees of hypertrophy (see Results). Sham animals had the same surgery without banding of the aorta. 10 to $14 \mathrm{~d}$ before death, animals were randomly assigned either levo-thyroxine $\left(\mathrm{T}_{4}, 500 \mu \mathrm{g} / \mathrm{kg}\right.$ per day; Sigma Chemical Co., St. Louis, MO) or vehicle (saline containing $1 \mu \mathrm{mol} / \mathrm{liter}$ $\mathrm{NaOH}$ ) by daily intraperitoneal injection.

The degree of hypertrophy was assessed in two ways. Myocyte volume, an index of cell size (see below), was quantified in experiments where isovolumic perfusion was followed by myocyte isolation for assays of protein expression. LV dry weight, an index of heart mass, was measured in another series of experiments, after isovolumic perfusion and drying at $80^{\circ} \mathrm{C}$ for $48 \mathrm{~h}$.

The animals were maintained in accordance with the guidelines of the University of California Animal Use Committee and Guidelines for the Care and the Use of Laboratory Animals of the Institute of Laboratory Animal Resources, National Council (DHHS publication, National Institutes of Health [NIH] 85-23, 1985).

Isovolumic heart preparation for assessing $L V$ function and cytosolic $\left[\mathrm{Ca}^{2+}\right]$. The isovolumic heart preparation has been described in detail previously $(19,20)$. Isolated hearts were perfused using a KrebsHenseleit (KH) buffer containing (mmol/liter): $\mathrm{NaCl}(118), \mathrm{KCl}(6.0)$, $\mathrm{MgSO}_{4}$ (1.2), $\mathrm{CaCl}_{2}(2.0), \mathrm{NaHCO}_{3}$ (25), pyruvate (5.0), glucose (4.0), insulin $20 \mathrm{U} /$ liter and $95 \% \mathrm{O}_{2}-5 \% \mathrm{CO}_{2}\left(37^{\circ} \mathrm{C}\right.$, pH of 7.35-7.45). Hearts were paced at $5 \mathrm{~Hz}$. LV pressure was measured using a highfidelity transducer (Millar Instruments, Inc., Houston, TX) attached to a compliant latex balloon. LV end-diastolic pressure was set at 10 $\mathrm{mmHg}$ by inflating the balloon with saline. This end-diastolic pressure resulted in maximum LV developed pressure in all hearts. Balloon volumes were not measured. To achieve equal rates of coronary flow per gram LV weight, coronary perfusion pressure was set at 80 $\mathrm{mmHg}$ for sham hearts and $100 \mathrm{mmHg}$ for left ventricle hypertrophy (LVH) hearts (19). Myocardial relaxation was quantified by calculating the time constant of monoexponential pressure decline $\left(\tau_{\mathrm{P}}\right)$, assuming a nonzero asymptote. Minimum and maximum $\mathrm{dP} / \mathrm{dt}$ were determined from the difference in LV pressure between two data points with a time resolution of $2 \mathrm{~ms}$.

Cytosolic $\left[\mathrm{Ca}^{2+}\right]$ was measured in the epicardial surface of hearts using indo-1 fluorescence as described previously in detail (19-22). Potential sources of artifact were accounted for and/or minimized (heart motion [23], autofluorescence [21, 22] unhydrolyzed indo-1 AM [24], tissue filter effect [21, 22], and potential loading of indo-1 into endothelial cells or noncytosolic compartments [25, 26]). Hearts were loaded by coronary perfusion with the acetoxymethyl ester form of indo- 1 and excited at $350 \mathrm{~nm}$. The ratio of indo- 1 fluorescence at 385 and $456 \mathrm{~nm}$ was calibrated to calculate cytosolic $\left[\mathrm{Ca}^{2+}\right]$ (21). Fluorescence data were digitized with $2 \mathrm{~ms}$ time resolution and five consecutive transients were averaged to improve signal-to-noise ratio. The rate of $\left[\mathrm{Ca}^{2+}\right]$ transient decline $\left(\tau_{\mathrm{Ca}}\right)$ was calculated by fitting the monoexponential portion of $\left[\mathrm{Ca}^{2+}\right]$ decline (i.e, from $70-20 \%$ of the transient amplitude) assuming a nonzero asymptote.

Myocyte isolation and preparation of myocyte homogenates. After hemodynamic and fluorescence measurements, myocytes were isolated with collagenase (27). $\mathrm{KH}$ buffer, without added $\mathrm{Ca}^{2+}$ and supplemented with $5 \mathrm{mmol} / \mathrm{liter}$ Hepes and $0.5 \mathrm{mg} / \mathrm{ml}$ bovine albumin, was perfused for 10-15 min, then $1 \mathrm{mg} / \mathrm{ml}$ collagenase B (Boehringer Mannheim Corp., Indianapolis, IN) and $\mathrm{CaCl}_{2}$ to a final concentration of $40 \mu \mathrm{mol} /$ liter were added. After 30 to $45 \mathrm{~min}$, the ventricles were minced in Kraftbruhe medium at pH 7.2. Myocytes and nonmyocytes were separated by density centrifugation ( $5 \mathrm{~min}$ at $40 \mathrm{rpm}$ in $4 \%$ Ficoll-400) (28).

Myocyte number and volume were measured using a Coulter multisizer (Coulter Electronics, Inc., Hialeah, FL) as described previously by Gerdes, et al. (28). The mean number of cardiac myocytes isolated from hypertrophied and control hearts was similar $(8.8 \pm 2.1$ vs. $9.0 \pm 1.0 \times 10^{6} ; P=\mathrm{NS}$ ). More than $90 \%$ of the myocytes were rod shaped and calcium tolerant; hypercontracted myocytes and nonmyocytes were $<5 \%$ of the total cell volume.

Total myocyte homogenates were made by sonication in a phosphate-based buffer ( $\mathrm{pH} \mathrm{7.4)}$ containing protease inhibitors $(2 \mathrm{mmol} /$ liter EDTA, $0.5 \mathrm{mmol} / \mathrm{liter}$ PMSF, $2 \mu \mathrm{g} / \mathrm{ml}$ leupeptin, $2 \mu \mathrm{g} / \mathrm{ml}$ aprotinin). Disruption of myocyte membranes was confirmed under a microscope. Homogenates were snap frozen in an ethanol/dry ice bath and stored at $-70^{\circ} \mathrm{C}$ until use.

Western analysis for SERCA and phospholamban. Homogenate equivalent to 500 myocytes $(\sim 5 \mu \mathrm{g}$ protein) from a single heart was loaded into each well of an $0.1 \%$ SDS-polyacrylamide gel (10\% for SERCA, $15 \%$ for phospholamban) and separated on a minigel apparatus (Mini-PROTEAN II, Bio-Rad, Hercules, CA) for $\sim 60 \mathrm{~min}$ at $200 \mathrm{~V}$. Proteins were transferred electrophoretically to a polyvinylidene difluoride membrane, and gels were stained with Coomassie blue to document efficient transfer. Membranes were blocked (5\% wt/vol bovine albumin in Tris-based saline) then incubated with a polyclonal antibody against rabbit cardiac SERCA (29), or a monoclonal antibody against canine cardiac phospholamban (30) using a 1:1000 dilution (generous gifts from Drs. J. Lytton and J. Wang, respectively, University of Calgary, Calgary, Alberta, Canada). After incubation with ${ }^{125}$ I-labeled secondary antibodies and autoradiography, band densities were quantified in arbitrary units by scanning (Onescanner; Apple Computer Inc. and Ofoto ${ }^{\circledR}$ 2.0; Light Source Computer Images, Inc., Berkeley, CA). Band density increased linearly with increasing amount of myocyte protein over the range used in this study, and indo-1 loading of the heart did not change SERCA or phospholamban band densities (data not shown).

The amount or content of SERCA and phospholamban per myocyte was estimated by dividing band density by 500 , the number of myocytes loaded into each well. To take into account differences in myocyte volume or size among hearts, the concentration of SERCA or phospholamban was calculated by dividing the protein content per myocyte by the myocyte volume in the same heart, that is, densitometric units per myocyte per median myocyte volume.

Analysis of myosin heavy chain isoforms. Isoforms of myosin heavy chain (MHC) were separated by SDS-PAGE and quantified by silver staining (31). Myocyte homogenates in $2 \times$ Laemmli buffer were incubated at $100^{\circ} \mathrm{C}$ for $5 \mathrm{~min}$, loaded onto a 0.75 - $\mathrm{mm}$ thick $0.1 \%$ SDS- $4 \%$ polyacrylamide gel, and separated for $24 \mathrm{~h}$ at $4^{\circ} \mathrm{C}$ with a constant $80 \mathrm{~V}$ (PROTEAN II xi; Bio-Rad). Gels were fixed, stained (BioRad Silver Stain Kit; Bio-Rad) and dried. Bands representing $\alpha-\mathrm{MHC}$ 
and $\beta$-MHC were quantified by densitometry, and the contents and concentrations of $\alpha-\mathrm{MHC}$ and $\beta-\mathrm{MHC}$ were estimated as for SERCA and phospholamban.

Statistical analysis. Values are mean \pm SEM. Groups were compared by ANOVA; a two-tailed unpaired Student $t$ test with Bonferroni correction was used for post hoc testing. Differences were considered significant at $P<0.05$. Linear regression was done using all individual data points in all groups, but the data are shown as group means in the figures.

\section{Results}

The main goal of these experiments was to test if thyroid hormone would improve cardiac function in hypertrophy induced by sustained pressure overload. After banding of the aorta for 10-22 wk, a duration found in preliminary studies to induce hypertrophy, rats were treated with $\mathrm{T}_{4}$ or vehicle for the final 10-14 d before death. Treatment allocation was randomized. A relatively large dose of $\mathrm{T}_{4}$ was used $(500 \mu \mathrm{g} / \mathrm{kg}$ per day), to maximize the chances of observing $\mathrm{T}_{4}$-induced changes over a short time. This dose was tolerated well; mortality was only 1 out of 15 banded rats receiving $\mathrm{T}_{4}$, compared with 4 out of 18 banded rats receiving saline $(P<0.05)$. Thyroid-treated rats did lose $6-7 \%$ of body weight during treatment, compared with a body weight gain of $2-4 \%$ in rats treated with saline $(P<$ 0.05; Table I).

Left ventricular function. LV function was assessed in isolated perfused hearts after 10-14 d of treatment with $\mathrm{T}_{4}$ or saline. Representative pressure tracings are shown in Fig. 1 (top row), and the data are summarized in Table I. Banded animals treated with saline were divided into two groups based on myocardial relaxation $\left(\tau_{\mathrm{P}}\right)$, because slowing of relaxation is an early manifestation of decompensated function in LVH (32). In the group called $\mathrm{LVH}_{\text {comp }}$, comprising $57 \%$ of the banded animals ( 8 of 14), relaxation was normal, defined as $\tau_{P}$ within two standard deviations of the mean of the sham-saline group
(Fig. 2 and Table I). In the other $43 \%$ of banded rats, the group called $\mathrm{LVH}_{\text {decomp }}$, relaxation was slowed, with mean $\tau_{\mathrm{P}}$ $40 \%$ higher than in sham-saline $(P<0.05$, Table I; Fig. 2). The $\mathrm{LVH}_{\text {decomp }}$ group also had abnormal contractility, measured as maximum $\mathrm{dP} / \mathrm{dt}$ and $\mathrm{LV}$ developed pressure normalized to $\mathrm{LV}$ weight; both of these indices were normal in the $\mathrm{LVH}_{\text {comp }}$ group. Thus systolic and diastolic function were abnormal in a subset of the banded animals. Abnormal function was not related to the degree of myocyte hypertrophy or the banding protocol (the shorter duration more severe and longer duration less severe banding caused decompensated LVH in 44 and $40 \%$ of hearts, respectively).

In banded animals treated with $\mathrm{T}_{4}$, relaxation was not slowed in any of the 14 hearts. This is in contrast with the $43 \%$ incidence of slowed relaxation in banded animals treated with saline (Fig. 2 and Table I). Indeed, mean $\tau_{P}$ in the banded animals treated with $\mathrm{T}_{4}$ was the same as that found in sham rats treated with $\mathrm{T}_{4}$, and was significantly faster than in both salinetreated LVH groups (Table I and Fig. 2). Systolic function in banded animals was also improved by $\mathrm{T}_{4}$; maximum $\mathrm{dP} / \mathrm{dt}$ in banded animals treated with $\mathrm{T}_{4}$ was similar to sham animals treated with $\mathrm{T}_{4}$, and was again greater than both saline-treated banded groups (Table I). Thus thyroid hormone treatment not only prevented the abnormalities in systolic and diastolic function seen in a subset of rats with chronic pressure overload hypertrophy, but also improved function in all banded animals.

Calcium handling. To test whether the changes in myocardial relaxation with banding and $\mathrm{T}_{4}$ were related to changes in myocyte calcium handling, cytosolic $\left[\mathrm{Ca}^{2+}\right]$ was determined simultaneously with LV pressure in perfused hearts. Representative tracings are in Fig. 1 (bottom row), and the data are summarized in Table I.

There were no differences in mean systolic or diastolic $\left[\mathrm{Ca}^{2+}\right]$ among the groups (Table I). However, the rate of decline of $\left[\mathrm{Ca}^{2+}\right]$, assessed by $\tau_{\mathrm{Ca}}$ (Fig. 1), varied in the groups

Table I. Effects of $T_{4}$ in Sham and Banded (LVH) Rats

\begin{tabular}{|c|c|c|c|c|c|}
\hline & Sham & $\mathrm{LVH}_{\text {comp }}$ & $\mathrm{LVH}_{\text {decomp }}$ & LVH & Sham \\
\hline Treatment & saline & saline & saline & $\mathrm{T}_{4}$ & $\mathrm{~T}_{4}$ \\
\hline Number & 7 & 8 & 6 & 14 & 7 \\
\hline Body weight (gram) & $407 \pm 14$ & $435 \pm 12$ & $445 \pm 19$ & $403 \pm 7^{\ddagger}$ & $403 \pm 8$ \\
\hline Body weight change during treatment (\%) & $+3 \pm 1$ & $+4 \pm 1$ & $+2 \pm 2$ & $-7 \pm 1^{\ddagger}$ & $-6 \pm 1 *$ \\
\hline DevP (mmHg) & $91 \pm 2$ & $124 \pm 4^{*}$ & $93 \pm 5$ & $148 \pm 5^{\ddagger}$ & $120 \pm 3^{*}$ \\
\hline DevP/LV dry weight $\left(\mathrm{mmHg} / \mathrm{mg} \times 10^{-2}\right)$ & $42 \pm 1$ & $39 \pm 1$ & $29 \pm 2 *$ & $44 \pm 2$ & ND \\
\hline $\mathrm{dP} / \mathrm{dt}_{\max }(\mathrm{mmHg} / \mathrm{s})$ & $3289 \pm 88$ & $3486 \pm 287$ & $2782 \pm 266^{*}$ & $4552 \pm 135^{\ddagger}$ & $4350 \pm 61 *$ \\
\hline$\tau_{\mathrm{P}}(\mathrm{ms})$ & $20 \pm 1$ & $21 \pm 1$ & $28 \pm 2^{*}$ & $16 \pm 1^{\ddagger}$ & $16 \pm 1 *$ \\
\hline$\tau_{\mathrm{Ca}}(\mathrm{ms})$ & $38 \pm 1$ & $41 \pm 3$ & $49 \pm 3^{*}$ & $33 \pm 3^{\ddagger}$ & $27 \pm 1 *$ \\
\hline Diastolic $\left[\mathrm{Ca}^{2+}\right]$ (nmol/liter) & $161 \pm 13$ & $172 \pm 16$ & $196 \pm 35$ & $208 \pm 20$ & $165 \pm 11$ \\
\hline Systolic $\left[\mathrm{Ca}^{2+}\right]$ (nmol/liter) & $1072 \pm 79$ & $1085 \pm 82$ & $997 \pm 91$ & $1107 \pm 57$ & $1286 \pm 77$ \\
\hline Myocyte volume $\left(10^{3} \mu \mathrm{m}^{3}\right)$ & $31 \pm 1$ & $39 \pm 1 *$ & $41 \pm 1 *$ & $44 \pm 1 *$ & $37 \pm 1 *$ \\
\hline LV dry weight (mg) & $216 \pm 6$ & \multicolumn{2}{|c|}{$315 \pm 9 *$} & $334 \pm 13^{*}$ & ND \\
\hline RV dry weight (mg) & $61 \pm 4$ & \multicolumn{2}{|c|}{$79 \pm 189$} & $84 \pm 30$ & ND \\
\hline
\end{tabular}

LVH was induced by banding of the aorta for 10-22 wk; rats were then treated with $\mathrm{T}_{4}$ or saline for 10-14 d. Isovolumic LV pressure and free cystosolic $\left[\mathrm{Ca}^{2+}\right]$ (indo-1) were measured in perfused hearts, then myocytes were isolated by collagenase digestion to quantify median myocyte volume using a Coulter-multisizer. Banded hearts treated with saline were categorized as $\mathrm{LVH}_{\text {comp }}$ or $\mathrm{LVH}_{\text {decomp }}$ based on whether isovolumic relaxation was normal or slowed (normal or increased $\tau_{\mathrm{P}}$, respectively). LV dry weight was determined in hearts that did not undergo collagenase digestion $(n=5$ for each; there was no difference between $\mathrm{LVH}_{\text {comp }}$ and $\mathrm{LVH}_{\text {decomp }}$ so data were combined). DevP was normalized by the mean values of $\mathrm{LV}$ dry weight. Values are mean \pm SEM. $* P<0.05$ vs. sham-saline; ${ }^{\ddagger} P<0.05$ vs. $\mathrm{LVH}_{\text {comp }}$ and $\mathrm{LVH}_{\text {decomp. }}$. 


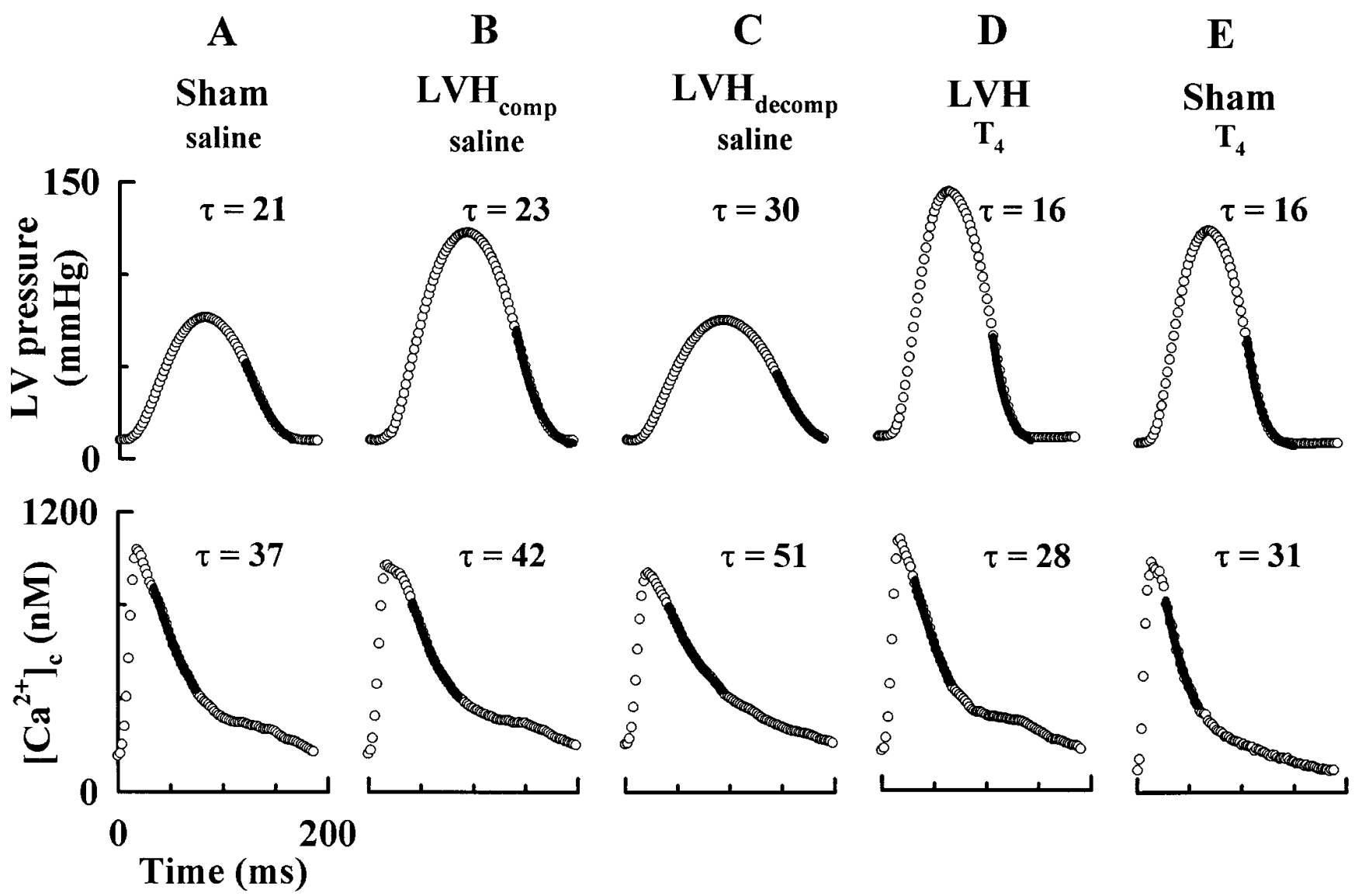

Figure 1. Effects of banding and $\mathrm{T}_{4}$ on isovolumic $\mathrm{LV}$ pressure and cytosolic $\left[\mathrm{Ca}^{2+}\right]$ transients. Tracings are representative of hearts in each of the groups in Table I. The open circles are individual data points, the solid lines are the fits used to calculate the time constants of exponential LV pressure and cytosolic $\left[\mathrm{Ca}^{2+}\right]$ decline $\left(\tau_{\mathrm{P}}\right.$ and $\tau_{\mathrm{Ca}}$, respectively). $(A)$ sham animal treated with vehicle $($ saline $) ;(B)$ banded animal treated with saline that had normal function $\left(\mathrm{LVH}_{\text {comp }}\right) ;(C)$ saline-treated banded animal with abnormal function $\left(\mathrm{LVH}_{\text {decomp }}\right) ;(D)$ banded animal treated with $\mathrm{T}_{4} ;(E)$ sham treated with $\mathrm{T}_{4}$.

similar to $\tau_{\mathrm{P}}$. Mean $\tau_{\mathrm{Ca}}$ of the $\mathrm{LVH}_{\text {comp }}$ group treated with saline was not significantly different from sham-saline, whereas $\tau_{\mathrm{Ca}}$ was $29 \%$ higher in the $\mathrm{LVH}_{\text {decomp }}$ group $(P<0.05)$. In banded animals treated with $\mathrm{T}_{4}, \tau_{\mathrm{Ca}}$ was $20-33 \%$ lower than in banded groups treated with saline (Table I), indicating that the

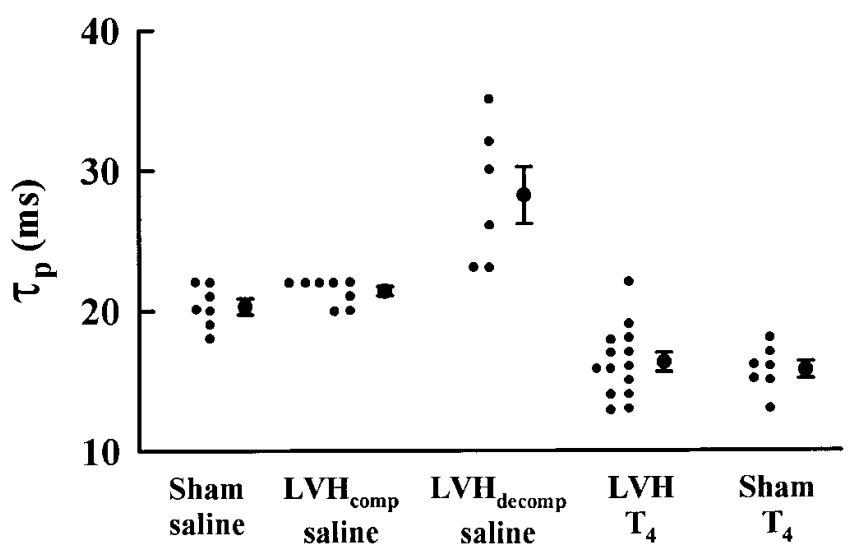

Figure 2. Thyroid improved relaxation in banded hearts. The $\tau_{\mathrm{P}}$ of every heart and the mean values of each group are shown (means as in Table I). rate calcium decline was faster. Calcium decline was also faster in normal (sham) hearts treated with $\mathrm{T}_{4}$ (Table $\mathrm{I}$ ), in agreement with prior reports $(10,11)$.

When $\tau_{\mathrm{P}}$ and $\tau_{\mathrm{Ca}}$ in all 42 hearts in the five groups were analyzed by linear regression (Fig. 3), a strong positive correlation was observed $(r=0.61, P<0.01)$. These data provide the first direct support for the idea that slowing of the decline of cytosolic calcium is an important factor in slowing myocardial relaxation in LVH. Furthermore, it also suggests that faster relaxation in thyroid-treated hearts was due to faster decline of $\left[\mathrm{Ca}^{2+}\right]$.

Myocyte proteins. We next tested whether the abnormalities in function and myocyte calcium handling with banding, and the improvements with thyroid hormone, were related to changes in myocyte proteins. After pressure and calcium measurements, myocytes were isolated, and median myocyte volume from each heart was measured using a Coulter multisizer. Unfractionated myocyte homogenates were used to quantify SERCA, phospholamban, and the isoforms of $\operatorname{MHC}(\alpha$ and $\beta)$.

The content of SERCA per myocyte was increased significantly in the $\mathrm{LVH}_{\text {comp }}$ group ( $22 \%$ vs. sham-saline, $P<0.05$, Fig. 4), indicating a net SERCA induction. However, median myocyte volume also increased in the $\mathrm{LVH}_{\text {comp }}$ group (26\% vs. sham-saline, $P<0.05$, Table I), so that the overall result was no change in the myocyte concentration of SERCA. In con- 


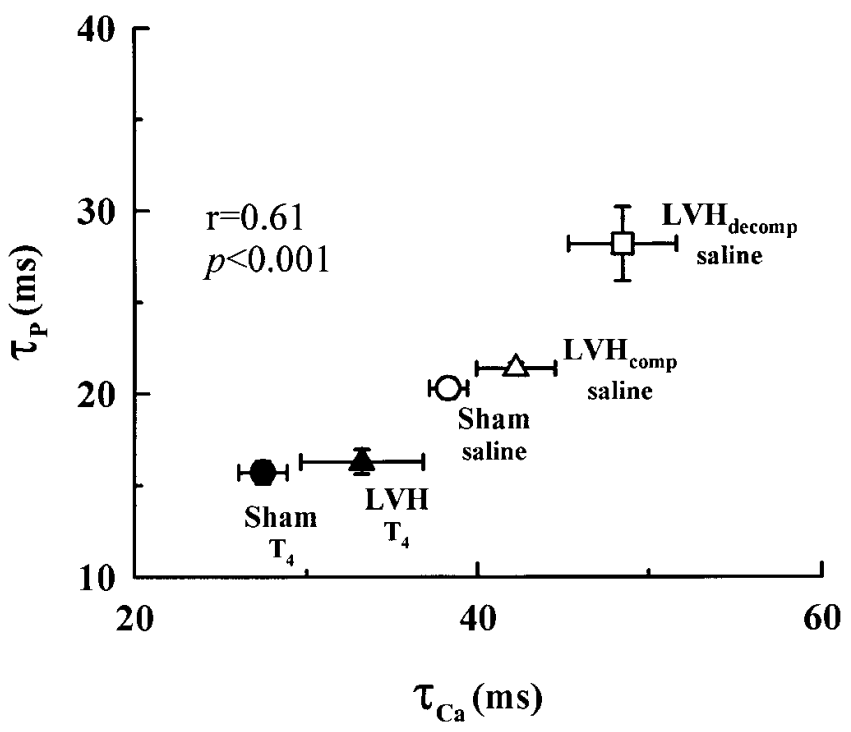

Figure 3. $\tau_{\mathrm{P}}$ was related to $\tau_{\mathrm{Ca}}$ in all hearts in all five groups. $\tau_{\mathrm{P}}$ and $\tau_{\mathrm{Ca}}$ from all 42 individual hearts were used to calculate the linear regression. For clarity, only mean $\tau_{\mathrm{Ca}}$ and $\tau_{\mathrm{P}}$ of the groups are shown. It was not possible to determine whether a more complicated model (e.g., exponential, two-phase) would provide a better fit between $\tau_{\mathrm{Ca}}$ and $\tau_{\mathrm{P}}$ because of variability in the data.

trast, in the $\mathrm{LVH}_{\text {decomp }}$ group of banded animals treated with saline, SERCA content per myocyte failed to increase. Since SERCA content did not change but myocyte volume increased significantly ( $32 \%$ vs. sham-saline, $P<0.05$, Table I), the net effect was a significant decrease in the myocyte concentration of SERCA in the $\mathrm{LVH}_{\text {decomp }}$ group (13\% lower than shamsaline, $P<0.05$, Fig. 4 ).

Treatment of banded animals with $\mathrm{T}_{4}$ increased SERCA content (47 and $88 \%$ vs. $\mathrm{LVH}_{\text {comp }}$ and $\mathrm{LVH}_{\text {decomp }}$, respectively; $P<0.05$, Fig. 4). This increase in SERCA content with $\mathrm{T}_{4}$ was sufficient to produce an increase in SERCA concentration, even taking into account the large myocyte volume with banding plus $\mathrm{T}_{4}$ (Table I). Thus mean SERCA concentration in the LVH-T 4 group was increased 30 and $47 \%$ relative to $\mathrm{LVH}_{\text {comp }}$ and $\mathrm{LVH}_{\text {decomp }}$ groups, respectively $(P<0.05$, Fig. 4). In fact, the SERCA concentration in banded myocytes given $\mathrm{T}_{4}$ was at least as high as in sham rats treated with $\mathrm{T}_{4}$ (Fig. 4). To our knowledge, this is the first report that the concentration of SERCA protein in myocytes can be increased in hearts subjected to pressure overload.

There was an inverse relationship $(r=-0.48, P<0.01)$ between SERCA concentration and $\tau_{\mathrm{Ca}}$ in all 42 hearts in the five groups (Fig. 5). The relation was not as strong when SERCA content was related to $\tau_{\mathrm{Ca}}$. These data support the idea that SERCA transport of $\mathrm{Ca}^{2+}$ into the sarcoplasmic reticulum is a major factor determining the rate of $\left[\mathrm{Ca}^{2+}\right]$ decline (33) and suggest that the concentration of SERCA is a physiologically relevant index of protein amount. The data further indicate that changes in SERCA could account for the changes in calcium handling with decompensation and thyroid treatment in LVH.

Phospholamban is also involved in myocyte calcium handling, via inhibition of SERCA. However, phospholamban content and concentration were unchanged in all banded groups (Fig. 6). Interestingly, $\mathrm{T}_{4}$ reduced phospholamban content and concentration in sham animals, as reported previously (12), but this effect was not seen in banded rats (Fig. 6).

$\alpha-\mathrm{MHC}$ regulation in the groups was similar to SERCA (Fig. 7). The banded groups treated with saline differed in that $\alpha$-MHC content was increased significantly in hypertrophy with normal function $\left(\mathrm{LVH}_{\text {comp }}\right)$, but not in hypertrophy with abnormal function $\left(\mathrm{LVH}_{\text {decomp }}\right)$ (Fig. 7). Thyroid hormone treatment of banded animals had a robust effect to increase $\alpha-\mathrm{MHC}$ concentration (43 and $100 \%$ relative to $\mathrm{LVH}_{\text {comp }}$ and $\mathrm{LVH}_{\text {decomp }}$ groups, respectively, $P<0.01)$.

$\beta$-MHC concentration was increased significantly in both banded groups given saline $(70-115 \%$ relative to sham-saline, $P<0.001$ ), in agreement with prior reports. However, the combination of banding and thyroid had no further effect (Fig. 7).

The bottom panel of Fig. $7 B$ are the total MHC concentration in myocytes in the different groups (i.e., the sum of $\alpha$ - and $\beta$-MHC concentrations). There was no significant change in total $\mathrm{MHC}$ concentration in any group, although there was a tendency to a higher MHC concentration in the hypertrophied cells. To compare with previous reports $(15,18)$, the relative fraction of MHC that is $\alpha-\mathrm{MHC}$ is also shown. Because $\beta$-MHC concentration was not different in the banded groups (Fig. 7, middle panel), the reduced percentage of $\alpha$-MHC in pressure overload alone and the increased percent $\alpha$-MHC

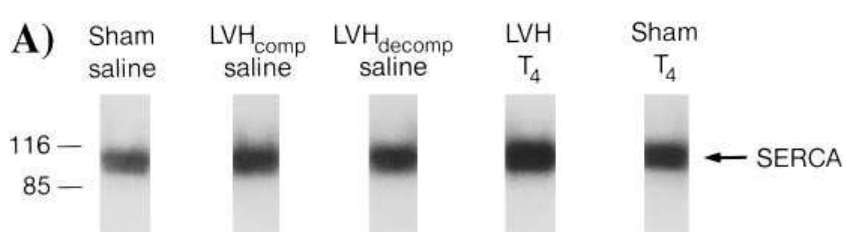

B)

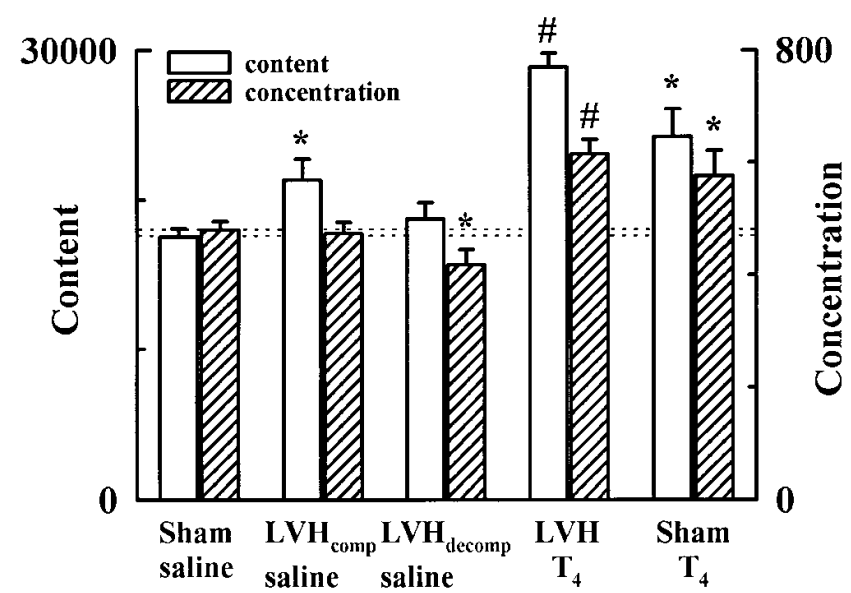

Figure 4. $\mathrm{T}_{4}$ increased myocyte SERCA concentration in banded animals. Panel $A$ is a representative autoradiograph, and Panel $B$ is a summary of the data. Total homogenates from 500 myocytes were loaded into each well, and Western analysis was done using a $10 \%$ SDS-polyacrylamide gel. Left margin in $A$ indicates molecular weight (kD). Data in $B$ are expressed both as myocyte content (open bars), in arbitrary density units per myocyte, and as myocyte concentration (striped bars), in density units per myocyte per median myocyte volume in cubic microns (volume as in Table I). $* P<0.05$ vs. shamsaline; ${ }^{\#} P<0.05$ vs. $\mathrm{LVH}_{\text {comp }}$ and $\mathrm{LVH}_{\text {decomp. }}$. 


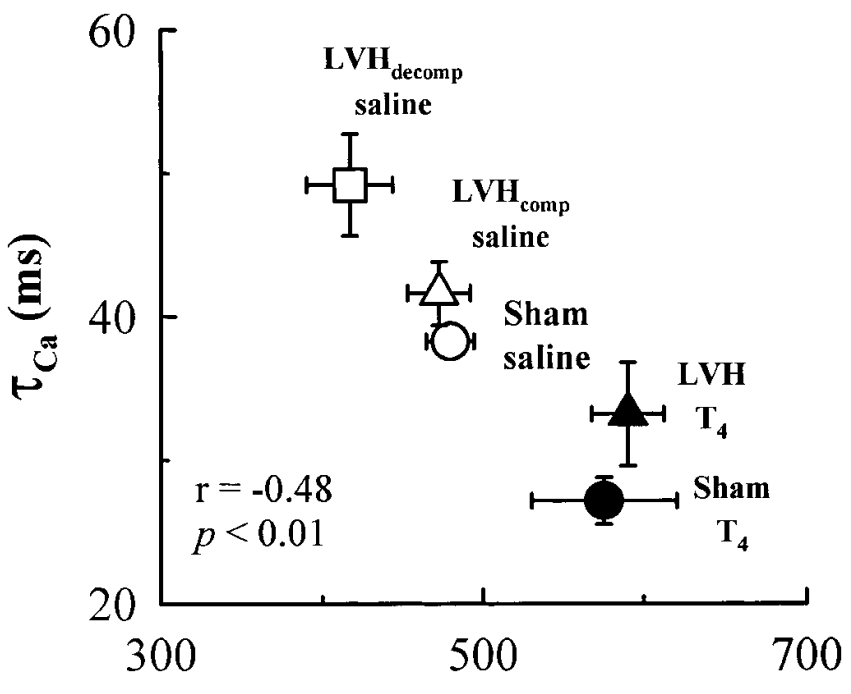

\section{SERCA Concentration}

Figure 5. $\tau_{\mathrm{Ca}}$ was related to myocyte SERCA concentration in all hearts in all groups. Data from all 42 individual hearts were used to calculate the linear regression, but only mean values of SERCA concentration and $\tau_{\mathrm{Ca}}$ are shown for clarity.

with thyroid hormone can both be explained by changes in the levels of $\alpha$-MHC (Fig. $7 \mathrm{~B}$, top panel). Thus pathological hypertrophy might be best characterized by failure of $\alpha$-MHC induction, more than by preferential $\beta$-MHC up-regulation. In light of prior work $(18,34)$, the percentage of $\beta$-MHC protein concentration in sham- $\mathrm{T}_{4}$ group $(29 \%)$ was somewhat higher than anticipated. We also did not see a decrease in the absolute $\beta$-MHC content or concentration with thyroid in any group (Fig. 7). Methodological differences from previous studies may account for some of these findings (e.g., we determined absolute amounts of $\beta$-MHC protein in isolated myocyte homogenates, rather that relative amounts in tissue homogenates).

\section{Discussion}

The key finding of this study is that thyroid hormone improves LV function in a model of chronic, sustained pressure overload. In addition, the functional benefit is related to effects of thyroid on calcium handling and gene expression of cardiac myocytes. These effects of thyroid hormone are similar to the physiological hypertrophy induced by exercise. Thus induction of physiological hypertrophy by thyroid hormone-like signaling may be a therapeutic strategy for treating cardiac dysfunction in pathological hypertrophy and heart failure.

We used a model of moderate-to-severe LVH (19) in which cardiac function was abnormal in $43 \%$ of the hearts treated with saline (vehicle). Similar variability in the response to pressure overload has been seen by others and ascribed to abnormal growth regulation $(35,36)$. In our study, thyroid hormone enhanced cardiac function in banded animals $\left(\mathrm{LVH}_{-} \mathrm{T}_{4}\right.$ group) compared with vehicle-treated controls $\left(\mathrm{LVH}_{\text {comp }}\right.$ and $\mathrm{LVH}_{\text {decomp }}$ groups). In fact, there was no evidence of abnormal cardiac function in banded animals that received $\mathrm{T}_{4}$. Because animals were randomly assigned to receive $T_{4}$ or vehicle, it is reasonable to assume that $\sim 43 \%$ of $\mathrm{LVH}^{-\mathrm{T}_{4}}$ group would have had abnormal cardiac function dysfunction without $\mathrm{T}_{4}$. However, the functional status of each heart was not known before treatment.

The rationale for using thyroid hormone to treat cardiac dysfunction in LVH is based on previous observations that thyroid hormone enhances function in normal hearts (10-13) and in the postinfarction model of heart failure (14-17). However, there are differences in the current study that provide new and complementary information. First, thyroid hormone may enhance cardiac function in the postinfarct model by decreasing arterial resistance, which reduces pressure overload $(13,16)$. Therefore, we studied the effects of thyroid hormone in animals with aortic banding, where the degree of pathological stress (pressure overload) was less likely to be reduced by $\mathrm{T}_{4}$. Second, physiology and protein expression of noninfarcted myocardium in the postinfarct model may be different from the pressure overload model. Third, we evaluated cardiac function, cytosolic $\left[\mathrm{Ca}^{2+}\right]$, and the myocyte concentration of contractile and calcium-handling proteins in the same hearts.

The changes in myocytes gene expression seen in our study are potentially causative for changes in cardiac function. SERCA concentration in isolated cardiac myocytes was closely related to the rate of decline of the calcium transient (i.e., $\tau_{\mathrm{Ca}}$ ). This relationship held over a range of $\tau_{\mathrm{Ca}}$ both above and below normal in all groups. Furthermore, $\tau_{\mathrm{Ca}}$ in turn correlated well with the rate of pressure decline $\left(\tau_{\mathrm{P}}\right)$ in the isolated isovolumic heart, again over a range of $\tau_{\mathrm{P}}$. These results are the first direct evidence that SERCA concentration in myocytes is a determinant

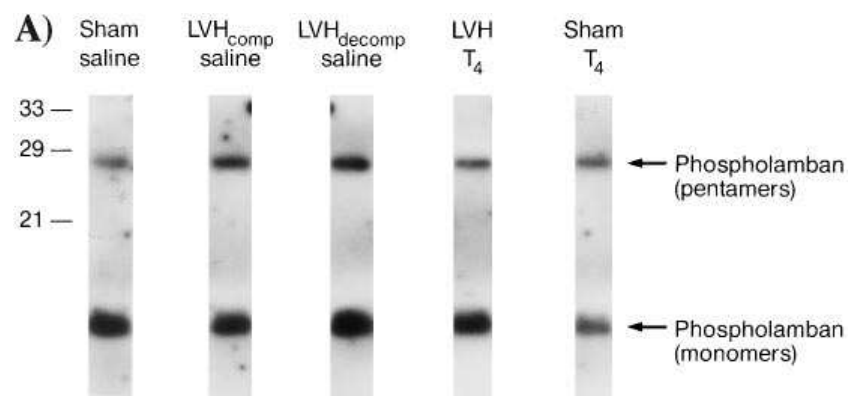

B)

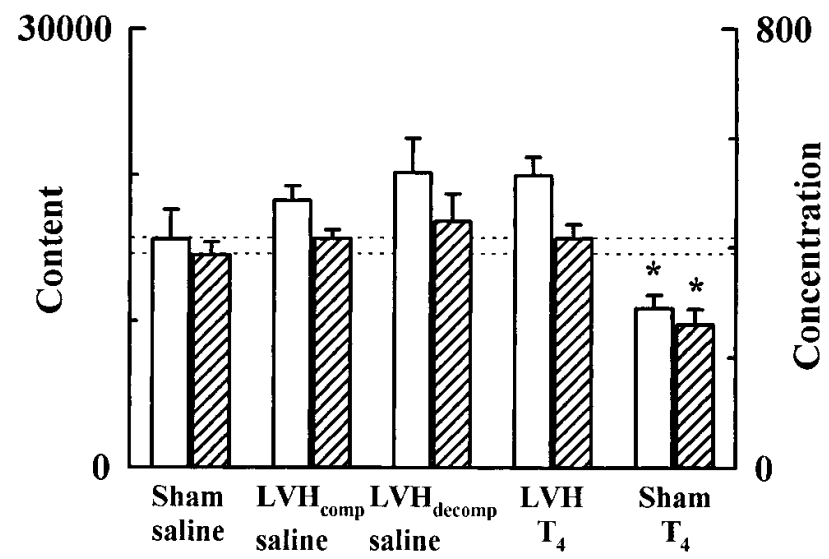

Figure 6. $\mathrm{T}_{4}$ had no effect on phospholamban in banded animals. As in Fig. 4, except a $15 \%$ SDS gel was used. $* P<0.05$ vs. sham-saline. 


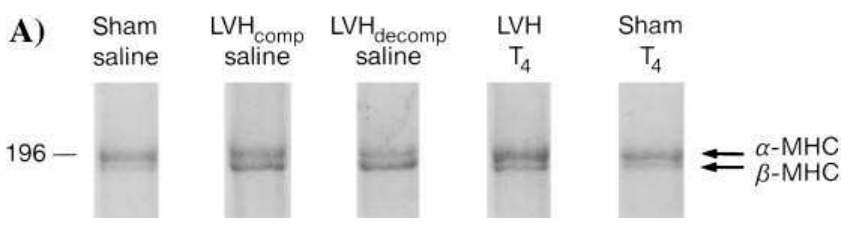

B)

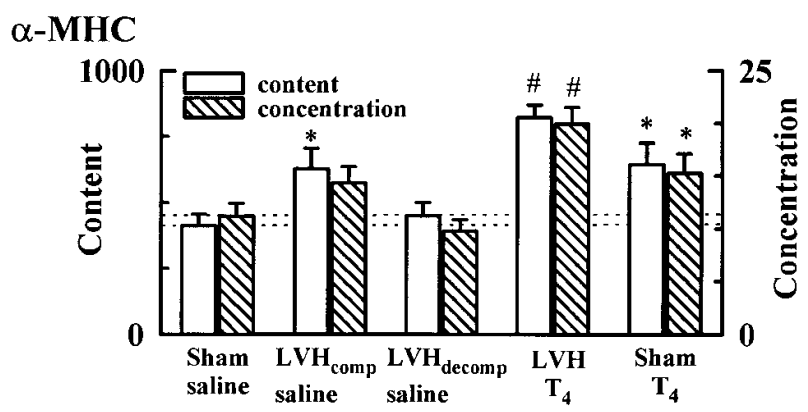

$\beta$-MHC

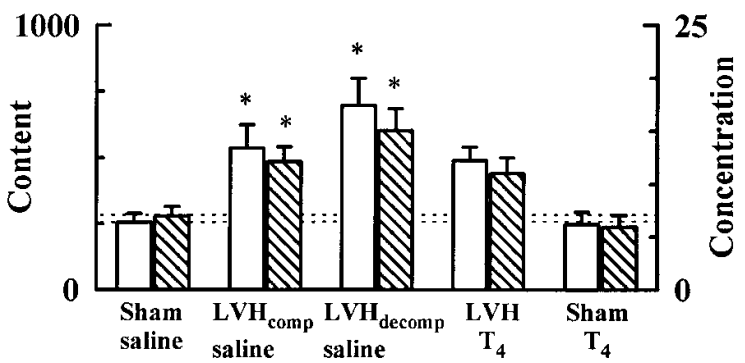

Total MHC ( $\alpha \%)$

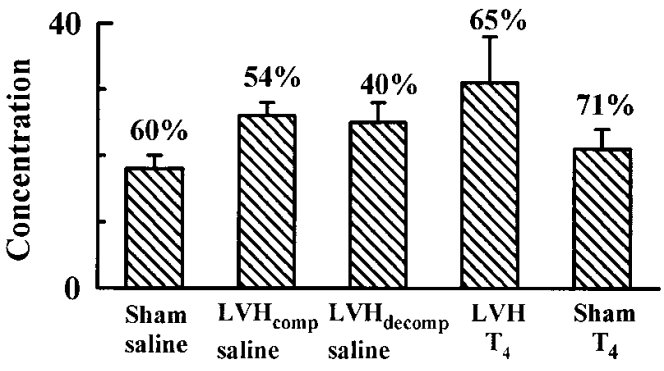

Figure 7. $\mathrm{T}_{4}$ increased $\alpha$-MHC protein concentration in myocytes from banded rats. As in Fig. 4, except a 4\% SDS-polyacrylamide gel was used, and $\alpha$ - and $\beta$-MHC were detected by silver staining. The left margin in panel $A$ indicates molecular weight (kD). In panel $B$, open bars are content and stippled bars are concentration. The total MHC bars (bottom) are the sum of the $\alpha$ - and $\beta$-MHC concentrations, and the percent of this total which is $\alpha$-MHC is listed. $* P<0.05$ vs. sham-saline; ${ }^{\#} P<0.05$ vs. $\mathrm{LVH}_{\text {comp }}$ and $\mathrm{LVH}_{\text {decomp. }}$.

of myocardial relaxation. They also support the idea that decreased SERCA in LVH and heart failure might impair relaxation (37-39), and further suggest that increased SERCA can improve relaxation. Relaxation in the banded group treated with thyroid may also have been enhanced by the increase of $\alpha-\mathrm{MHC}$ concentration in myocytes. $\alpha$-MHC has a faster rate of crossbridge cycling than $\beta$-MHC so that a sarcomere with a higher proportion of $\alpha$-MHC should have faster relaxation (40). Indeed, the banded group treated with thyroid had a higher proportion of $\alpha$-MHC, due to increased $\alpha-\mathrm{MHC}$ with no change in $\beta$-MHC.
Although the expression of SERCA and $\alpha$-MHC proteins were related to calcium handling and function, the degree of myocyte hypertrophy was not. Myocyte volume was not significantly different in $\mathrm{LVH}_{\text {comp }}$ and $\mathrm{LVH}_{\text {decomp }}$ groups, even though function and calcium handling were different. Furthermore, the combination of pressure overload and thyroid hormone in the $\mathrm{LVH}-\mathrm{T}_{4}$ group resulted in only a trend toward increased myocyte size or LV mass relative to pressure overload alone. Thus the degree of myocyte hypertrophy was not related to cardiac function, calcium handling, or SERCA and MHC expression. This is in agreement with previous work that dissociated mRNA levels of SERCA and $\alpha$-MHC from overall cardiac growth (41). These investigators found that unloaded rat hearts (transplanted onto the abdominal aorta of recipients) had increased SERCA and $\alpha$-MHC mRNA in response to thyroid without an increase of cardiac weight. Thus, there is increasing evidence that gene expression can be modulated independently from overall cardiac growth.

Could the beneficial effects of thyroid be exploited therapeutically? Thyroid is thought to signal both through nuclear receptors and by less-defined extranuclear mechanisms (13). The action of nuclear receptors to increase transcription can likely explain the induction of SERCA and $\alpha-\mathrm{MHC}$, at least in part, based on extensive work by others $(18,41-44)$ but we did not measure mRNA levels. It is also likely that other genes were induced by thyroid in this study, such as the sarcolemmal $\mathrm{Na}^{+}-\mathrm{K}^{+}$ATPase, $\beta$-adrenergic receptors, and enzymes of oxidative phosphorylation (13). To the extent that thyroid signaling is selective for specific genes or groups of genes, it might be possible to design drugs that have more favorable effects and fewer undesirable ones, such as the weight loss observed in this study. Indeed a thyroid hormone analog is being tested that has fewer systemic effects and tachycardia (45).

Thyroid signaling might have some advantages relative to other growth factors being tested in cardiac disease. Growth hormone and insulin-like growth factor-I increase contractility in animal models $(46,47)$ and humans $(48)$, but may also slow relaxation and induce cardiac fibrosis (49). In contrast, thyroid hormone enhances relaxation and is not thought to activate fibroblasts (50). Further study is needed to determine the best way to stimulate cardiac growth as treatment for heart failure.

\section{Acknowledgments}

We thank Anthony Baker, Ph.D., Jessica Halow, Ph.D., Carlin S. Long, M.D., and Barry Massie, M.D., for helpful discussions and review of the manuscript, and Gunnard Modin for doing the statistics.

Kevin C. Chang is a recipient of the National Research Service Award from the National Institutes of Health (NIH) (HL-09064). This work was supported in part by grants from the NIH, K08 HL02448 and R01 HL-54890 (S.A. Camacho), K08 HL-02883 (V.M. Figueredo), RO1 DK33923 (M.W. Weiner), RO1 HL42150, and RO1 HL31113 (P.C. Simpson); American Heart Association, California Affiliate Grant-in-Aid 95-220 (S.A. Camacho), 94-211 (V.M. Figueredo) and National Grant-in-Aid 94-6930 (S.A. Camacho); and the Research Service of the Department of Veterans Affairs (M.W. Weiner and P.C. Simpson).

\section{References}

1. Cohn, J.N., M.R. Bristow, K.R. Chien, W.S. Colucci, O.H. Frazier, L.A Leinwand, B.H. Lorell, A.J. Moss, E.H. Sonnenblick, R.A. Walsh, et al. 1997. Report of the National Heart, Lung, and Blood Institute Special Emphasis Panel on Heart Failure Research. Circulation. 95:766-770. 
2. Bing, O.H., W.W. Brooks, C.H. Conrad, S. Sen, C.L. Perreault, and J.P. Morgan. 1991. Intracellular calcium transients in myocardium from spontaneously hypertensive rats during the transition to heart failure. Circ. Res. 68:13801400 .

3. Feldman, A.M., E.O. Weinberg, P.E. Ray, and B.H. Lorell. 1993. Selective changes in cardiac gene expression during compensated hypertrophy and the transition to cardiac decompensation in rats with chronic aortic banding. Circ. Res. 73:184-192.

4. Scheuer, J., and P.M. Buttrick. 1987. The cardiac hypertrophic responses to pathologic and physiologic loads. Circulation. 75:163-168.

5. Malhotra, A., S. Penpargdul, T.F. Schaible, and J. Scheuer. 1981. Contractile proteins and sarcoplasmic reticulum in physiologic cardiac hypertrophy. Am. J. Physiol. 24:H263-H267.

6. Schaible, T.F., and J. Scheuer. 1985. Cardiac adaptations to chronic exercise. Prog. Cardiovasc. Dis. 27:297-324.

7. Schaible, T.F., G.J. Ciambrone, J.M. Capasso, and J. Scheuer. 1984. Cardiac conditioning ameliorates cardiac dysfunction associated with renal hypertension in rats. J. Clin. Invest. 73:1086-1094.

8. Schaible, T.F., A. Malhotra, G.J. Ciambrone, and J. Scheuer. 1986. Chronic swimming reverses cardiac dysfunction and myosin abnormalities in hypertensive rats. J. Appl. Physiol. 60:1435-1441.

9. Buttrick, P.M., A. Malhotra, and J. Scheuer. 1988. Effects of systolic overload and swim training on cardiac mechanics and biochemistry in rats. $J$. Appl. Physiol. 64:1466-1471.

10. MacKinnon, R., J.K. Gwathmey, P.D. Allen, G.M. Briggs, and J.P. Morgan. 1988. Modulation by the thyroid state of intracellular calcium and contractility in ferret ventricular muscle. Circ. Res. 63:1080-1089.

11. Bing, O.H.L., N.L. Hague, C.L. Perreault, C.H. Conrad, W.W. Brooks, S. Sen, and J.P. Morgan. 1994. Thyroid hormone effects on intracellular calcium and inotropic responses of rat ventricular myocardium. Am. J. Physiol. 267: H1112-H1121.

12. Kiss, E., G. Jakab, E.G. Kranias, and I. Edes. 1994. Thyroid hormoneinduced alterations in phospholamban protein expression: Regulatory effects on sarcoplasmic reticulum $\mathrm{Ca}^{2+}$ transport and myocardial relaxation. Circ. Res. 75:245-251.

13. Klemperer, J.D., K. Ojamaa, and I. Klein. 1996. Thyroid hormone therapy in cardiovascular disease. Prog. Cardiovasc. Dis. 38:329-336.

14. Gay, R.G., T.A. Gustafson, S. Goldman, and E. Morkin. 1987. Effects of L-thyroxine in rats with chronic heart failure after myocardial infarction. Am. J. Physiol. 253:H341-H346.

15. Gay, R.G., S. Graham, M. Aguirre, S. Goldman, and E. Morkin. 1988. Effects of 10- to 12-day treatment with L-thyroxine in rats with myocardial infarction. Am. J. Physiol. 255:H801-H806.

16. Morkin, E., G.D. Pennock, T.E. Raya, J.J. Bahl, and S. Goldman. 1993. Studies on the use of thyroid hormone and a thyroid hormone analogue in the treatment of congestive heart failure. Prog. Cardiovasc. Dis. 56:554-560.

17. Mahaffey, K.W., T.E. Raya, G.D. Pennock, E. Morkin, and S. Goldman. 1995. Left ventricular performance and remodeling in rabbits after myocardial infarction: effects of a thyroid hormone analogue. Circulation. 91:794-801.

18. Izumo, S., A. Lompre, M. Matsuoka, G. Koren, K. Schwartz, B. NadalGinard, and V. Mahdavi. 1987. Myosin heavy chain messenger RNA and protein isoform transitions during cardiac hypertrophy: interaction between hemodynamic and thyroid hormone-induced signals. J. Clin. Invest. 79:970-977.

19. Chang, K.C., J.H. Schreur, M.W. Weiner, and S.A. Camacho. 1996. Impaired $\mathrm{Ca}^{2+}$ handling is an early manifestation of pressure-overload hypertrophy in rat hearts. Am. J. Physiol. 271:H228-H234.

20. Camacho, S.A., R. Brandes, V.M. Figueredo, and M.W. Weiner. 1994. Calcium transient decline and myocardial relaxation are slowed during low flow ischemia in rat hearts. J. Clin. Invest. 93:951-957.

21. Brandes, R., V.M. Figueredo, S.A. Camacho, A.J. Baker, and M.W. Weiner. 1993. I. Quantitation of cytosolic $\left[\mathrm{Ca}^{2+}\right]$ in whole perfused rat hearts using indo-1 fluorometry. Biophys. J. 65:1973-1982.

22. Brandes, R., V.M. Figueredo, S.A. Camacho, A.J. Baker, and M.W. Weiner. 1993. II. Investigation of factors affecting fluorometric quantitation of cytosolic $\left[\mathrm{Ca}^{2+}\right]$ in perfused hearts. Biophys. J. 65:1983-1993.

23. Brandes, R., V.M. Figueredo, S.A. Camacho, B.M. Massie, and M.W. Weiner. 1992. Suppression of motion artifacts in fluorescence spectroscopy of perfused hearts. Am. J. Physiol. 263:H972-H980.

24. Figueredo, V.M., R. Brandes, M.W. Weiner, B.M. Massie, and S.A. Camacho. 1993. Endocardial versus epicardial differences of intracellular free calcium under normal and ischemic conditions in perfused rat hearts. Circ. Res. 72: 1082-1090.

25. Figueredo, V.M., R. Brandes, M.W. Weiner, B.M. Massie, and S.A. Camacho. 1992. Cardiac contractile dysfunction during mild coronary flow reductions is due to an altered calcium-pressure relationship in rat hearts. J. Clin. Invest. 90:1794-1802.

26. Schreur, J.H.M., V.M. Figueredo, M. Miyamae, D.M. Shames, A.J.
Baker, and S.A. Camacho. 1996. Cytosolic and mitochondrial $[\mathrm{Ca} 2+]$ in whole hearts using indo-1 AM: effects of high extracellular $\mathrm{Ca}^{2+}$. Biophys. J. 70:25712580 .

27. Isenberg, G., and U. Klockner. 1982. Calcium tolerant ventricular myocytes prepared by preincubation in a "KB Medium." Pflugers Arch. 395:6-18.

28. Gerdes, A.M., J.A. Moore, J.M. Hines, P.A. Kirkland, and S.P. Bishop. 1986. Regional differences in myocyte size in normal rat heart. Anat. Rec. 215: $420-426$.

29. Lytton, J., M. Westlin, S.E. Burk, G.E. Shull, and D.H. MacLennan. 1992. Functional comparisons between isoforms of the sarcoplasmic or endoplasmic reticulum family of calcium pumps. J. Biol. Chem. 267:14483-14489.

30. Morris, G.L., H.C. Cheng, J. Colyer, and J.H. Wang. 1991. Phospholamban regulation of cardiac sarcoplasmic reticulum $\mathrm{Ca}^{2+}-\mathrm{Mg}^{2+}$-ATPase. Mechanism of regulation and site of monoclonal antibody interaction. J. Biol. Chem. 266:11270-11275.

31. Esser, K.A., M.O. Boluyt, and T.P. White. 1988. Separation of cardiac myosin heavy chains by gradient SDS-PAGE. Am. J. Physiol. 255:H659-H663.

32. Lorell, B.H. 1991. Significance of diastolic dysfunction of the heart Annu. Rev. Med. 42:411-436.

33. Wier, W.G. 1990. Cytoplasmic $\left[\mathrm{Ca}^{2+}\right]$ in mammalian ventricle: dynamic control by cellular processes. Annu. Rev. Physiol. 52:467-485.

34. Ojamaa, K., J.D. Klemperer, S.S. MacGilvray, I. Klein, and A. Samarel. 1996. Thyroid hormone and hemodynamic regulation of b-myocin heavy chain promoter in the heart. Endocrinology. 137:802-808.

35. Koide, M., M. Nagatsu, M.R. Zile, M. Hamawaki, M.M. Swindle, G. Keech, G. DeFreyte, H. Tagawa, G.T. Cooper, and B.A. Carabello. 1997. Premorbid determinants of left ventricular dysfunction in a novel model of gradually induced pressure overload in the adult canine. Circulation. 95:1601-1610.

36. Ross, J., Jr. 1997. On variations in the cardiac hypertrophic response to pressure overload. Circulation. 95:1349-1351.

37. De La Bastie, D., D. Levitsky, L. Rappaport, J. Mercadier, F. Marotte, C. Wisnewsky, V. Brovkovich, K. Schwartz, and A. Lompre. 1990. Function of the SR and expression of its SERCA gene in pressure overload-induced cardiac hypertrophy in the rat. Circ. Res. 66:554-564.

38. Arai, M., H. Matsui, and M. Periasamy. 1994. Sarcoplasmic reticulum gene expression in cardiac hypertrophy and heart failure. Circ. Res. 74:555-564

39. Kiss, E., N.A. Ball, E.G. Kranias, and R.A. Walsh. 1995. Differential changes in cardiac phospholamban and sarcoplasmic reticular $\mathrm{Ca}^{2+}$-ATPase protein levels: effects on $\mathrm{Ca}^{2+}$ transport and mechanics in compensated pressure-overload hypertrophy and congestive heart failure. Circ. Res. 77:759-764.

40. Van Buren, P., D.E. Harris, N.R. Alpert, and D.M. Warshaw. 1995. Cardiac V1 and V3 myosins differ in their hydrolytic and mechanical activities in vitro. Circ. Res. 77:439-444.

41. Ojamaa, K., A.M. Samarel, J.M. Kupfer, C. Hong, and I. Klein. 1992. Thyroid hormone effects on cardiac gene expression independent of cardiac growth and protein synthesis. Am. J. Physiol. 263:E534-E540.

42. Rohrer, D.K., and W.H. Dillmann. 1988. Thyroid hormone markedly increases the mRNA coding for sarcoplasmic reticulum $\mathrm{Ca}^{2+}$-ATPase in the rat heart. J. Biol. Chem. 263:6941-6944.

43. Rohrer, D.K., R. Hartong, and W.H. Dillman. 1991. Influence of thyroid hormone and retinoic acid on slow sarcoplasmic reticulum $\mathrm{Ca}^{2+}$-ATPase and myosin heavy chain a gene expression in cardiac myocytes: delineation of Cis-active DNA elements that confer responsiveness to thyroid hormone but not to retinoic acid. J. Biol. Chem. 266:8638-8646.

44. Ojamaa, K., and I. Klein. 1991. Thyroid hormone regulation of alphamyosin heavy chain promoter activity assessed by in vivo DNA transfer in rat heart. Biochem. Biophys. Res. Commun. 179:1269-1275.

45. Pennock, G.D., T.E. Raya, J.J. Bahl, S. Goldman, and E. Morkin. 1993. Combination treatment with captopril and the thyroid hormone analogue 3,5 diiodothyropropionic acid: a new approach to improving left ventricular performance in heart failure. Circulation. 88:1289-1298.

46. Yang, R., S. Bunting, N. Gillett, R. Clark, and H. Jin. 1995. Growth hormone improves cardiac performance in experimental heart failure. Circulation. 92:262-267.

47. Duerr, R.L., S. Huang, H.R. Miraliakbar, R. Clark, K.R. Chien, and J. Ross, Jr. 1995. Insulin-like growth factor-1 enhances ventricular hypertrophy and function during the onset of experimental cardiac failure. J. Clin. Invest. 95: 619-627.

48. Fazio, S., D. Sabatini, B. Capaldo, C. Vigorito, A. Giordano, R. Guida, F. Pardo, B. Biondi, and L. Sacca. 1996. A preliminary study of growth hormone in the treatment of dilated cardiomyopathy. N. Engl. J. Med. 334:809-814.

49. Loh, E., and J.L. Swain. 1996. Growth hormone for heart failure: cause for cautious optimism. N. Engl. J. Med. 334:856-857.

50. Yao, J., and M. Eghbali. 1992. Decreased collagen gene expression and absence of fibrosis in thyroid hormone-induced myocardial hypertrophy. Response of cardiac fibroblasts to thyroid hormone in vitro. Circ. Res. 71:831-839. 